Check for updates

Cite this: RSC Adv., 2017, 7, 25512

\title{
Unidirectional anisotropy mediated giant memory effect in antiferromagnetic $\mathrm{Cr}_{2} \mathrm{O}_{3}$ nanorods
}

\author{
Ashish Chhaganlal Gandhi (D) and Sheng Yun Wu (D) * \\ Understanding the magnetic anisotropy of antiferromagnetic (AF) transition metal oxides is a matter of \\ importance for its future application in spintronics. In this study we have carried out temperature and \\ field-dependent magnetization measurements to investigate the magnetic anisotropy of $A F \mathrm{Cr}_{2} \mathrm{O}_{3}$ \\ nanorods (NRs) with $\langle d\rangle_{\mathrm{XRD}}=24 \mathrm{~nm}$ diameter. Our experimental findings suggest that the observed \\ field-cooled memory effect from exchange-coupled $\mathrm{Cr}_{2} \mathrm{O}_{3} \mathrm{NRs}$ is mediated by unidirectional anisotropy. \\ This finding opens an opportunity for devices utilizing the anisotropy field of bare $\mathrm{AF}_{\mathrm{Cr}_{2} \mathrm{O}_{3} \text { nano- }}$ \\ crystalline structures.
}

Received 6th April 2017

Accepted 8th May 2017

DOI: $10.1039 / \mathrm{c} 7 \mathrm{ra03934d}$

rsc.li/rsc-advances

resulting in an enhancement of the memory effect. ${ }^{22}$ However,

\section{Introduction}

In recent years one dimensional (1D) nanostructures with varied geometry such as nanowires, nanorods (NRs), nanotubes, and nanobelts have attracted much attention because of their promising technological applications in spintronics, lasers, generators, etc. ${ }^{1-3}$ The 1D nanostructured materials of antiferromagnetic (AF) transition metal oxides (TMOs), ${ }^{4,5}$ which show entirely different magnetic, optical and electrical properties from those of their bulk counterparts, have been used in the field of data storage ${ }^{6}$ and spin-valve devices. ${ }^{7}$ Depending on the finite size effect, the density of point defects (anionic and/or cationic vacancies) and surface effects, the magnetic properties of TMOs vary from spin-glass (SG), superparamagnetic (SPM) to ferromagnetic (FM). ${ }^{8-10}$ Among different TMOs, $\mathrm{Cr}_{2} \mathrm{O}_{3}$ is a low-anisotropy easy-axis AF with a Neel temperature around $\sim 307$ K. ${ }^{11}$ This material displays piezo magnetic, magneto electric effect, and also used in devices involving exchange bias (EB) effect make it promising material for future spintronic based applications. ${ }^{12-14}$ At nanoscale due to enhanced surface to volume ratio the interface interaction between surface spins and uncompensated core become more pronounced which has impulsed the fabrication and study of nano-systems looking for new properties and applications. ${ }^{10,15-18}$

The memory effect has been studied in superparamagnetic $(\mathrm{SPM})^{19}$ and $\mathrm{SG}^{20}$ system, in which formal shows weak fieldcooled (FC) aging and later shows aging with both FC and zero-field-cooled (ZFC). Recently, we have also reported memory effect from weakly interaction $\mathrm{Fe}_{3} \mathrm{O}_{4}$ nanoparticles. ${ }^{21}$ Using Monte Carlo simulation, it has been proved that interface exchange interaction in FM-core/AF-shell nanoparticles (NPs) provides an additive source for the frustration of the system

Department of Physics, National Dong Hwa University, Hualien 97401, Taiwan. E-mail: sywu@gms.ndhu.edu.tw unlike FM-core/AF-shell system, the interface interaction in bare AF nanostructures is much more complex. Very recently, we have reported spontaneous EB (SEB) from AF oxide NPs during the first field of hysteresis loop measurement. ${ }^{23}$ SEB in AF NPs is governed by the unidirectional anisotropy (UA) across frustrated surface spins and the uncompensated AF core mediated by interfacial strongly pinned spins. Such bare AF NPs exhibits field-cooled (FC) memory effect strength of which varies with the concentration of point defects resulting in disappearance in big size particles. ${ }^{8}$ In this work, we present detailed structural and magnetic characterization of $1 \mathrm{D} \mathrm{Cr}_{2} \mathrm{O}_{3}$ NRs. The aim is to study the effect of UA on the memory effect within the blocking state.

\section{Experimental details}

The as-received $\mathrm{CrO}_{2}$ NRs utilized in this study are commercially available NRs used in magnetic recording are annealed in an ambient atmosphere at $650{ }^{\circ} \mathrm{C}$ for 1 hour using a chemical vapor deposition (CVD) method, which resulted in formation of pure $\mathrm{Cr}_{2} \mathrm{O}_{3}$ NRs. The annealing of the sample is carried out in a tube furnace with heating rate of $5 \mathrm{~K} \mathrm{~min}^{-1}$ and allowed to cool down naturally. In general, $\mathrm{CrO}_{2}$ is a metastable oxide that readily decomposes into thermal dynamically stable chromia $\left(\mathrm{Cr}_{2} \mathrm{O}_{3}\right)$ as annealing temperature above $400{ }^{\circ} \mathrm{C}^{24,25}$ In the present work, the chemical reaction for the formation of chromia nanorods can be represented as $4 \mathrm{CrO}_{2} \rightarrow 2 \mathrm{Cr}_{2} \mathrm{O}_{3}+\mathrm{O}_{2}$, which is an antiferromagnetic insulator with a Néel temperature of $307 \mathrm{~K}^{26}$ And the small amount of $x$ indicates the existence of oxygen vacancies. The morphology and size distribution analysis was carried out by using field-emission scanning electron microscopy (FE-SEM, JEOL JSM-6500F) microscope. Structural properties were studied by using a high-resolution transmission electron microscopy (HR-TEM) 
and selective area diffraction pattern (SAED) using JEOL 2010 TEM working at $200 \mathrm{kV}$. Further analysis of structural property was carried out by synchrotron radiation X-ray diffraction (SRXRD) $(\lambda=0.7749 \AA)$ at National Synchrotron Radiation Research Center (NSRRC) beam line BL01C2 in Taiwan. The ZFC and FC temperature and field dependences of magnetization of the NRs were measured using a quantum design MPMSSQUID-VSM magnetometer. The magnetic memory effect on thermal variation of magnetization using FC-protocol with 100 Oe field is also investigated.

\section{Results and discussion}

\subsection{Morphological and stoichiometric analysis}

The SEM images of as-received $\mathrm{CrO}_{2}$ and $\mathrm{Cr}_{2} \mathrm{O}_{3}$ nanorods illustrated in Fig. 1(a) and (b) shows NRs like geometry having mean length around 100-200 nm, respectively. Fig. 1(c) and (d) depict the histogram of mean diameter distribution of NRs calculated from SEM images. The solid red line in Fig. 1(c) and (d) describes the asymmetric distribution of diameter fitted with log-normal distribution function: $f(d)=\frac{1}{\sqrt{2 \pi} d \sigma} \exp \left[-\frac{(\ln d-\ln \langle d\rangle)}{2 \sigma^{2}}\right]$, where $\langle d\rangle$ is the mean diameter and $\sigma$ standard deviation of the function. The fitted value of diameters and standard deviation are 24(1) $\mathrm{nm}$ and 0.25(2) for $\mathrm{CrO}_{2} \mathrm{NRs}$ and 35(1) nm and 0.22(3) for $\mathrm{Cr}_{2} \mathrm{O}_{3}$ NRs, respectively. The observed wide distribution and agglomeration behavior of the nanorods can significantly affect the magnetization properties. Fig. 2(a) and (b) show the TEM image of single $\mathrm{CrO}_{2}$ and $\mathrm{Cr}_{2} \mathrm{O}_{3}$ nanorod retaining crystalline structure. The inter-planar distance $d=0.3037$ and $0.3722 \mathrm{~nm}$ obtained from HR-TEM image for $\mathrm{CrO}_{2}$ and $\mathrm{Cr}_{2} \mathrm{O}_{3}$ nanorod, respectively, as shown in Fig. 2(c) and (d), corresponding to the (110) plane of $\mathrm{CrO}_{2}$ with $\mathrm{P4}_{2} / \mathrm{mnm}$ space group and the (200) plane of $\mathrm{Cr}_{2} \mathrm{O}_{3}$ with $R \overline{3} c$ space group, respectively. The SAED patterns of NRs displayed in Fig. 2(e) and (f) can be ascribed to the existence of tetragonal- $\mathrm{CrO}_{2}$ oriented along the [100] zone axis and hexagonal- $\mathrm{Cr}_{2} \mathrm{O}_{3}$ oriented along the $[-101]$ zone axis, respectively, revealing a single crystallinity.

The SR-XRD spectrum of NRs shown in Fig. 3 confirms the formation of pure $\mathrm{Cr}_{2} \mathrm{O}_{3}$ without any trace of $\mathrm{CrO}_{2}$ impurity. The observed broad SR-XRD peak of NRs could be fitted well with Gaussian function. The fitted value of full-width at halfmaximum (FWHM) of most intense (110) peak, indexed based on the space group $R \overline{3} c$ is $0.1566 \pm 0.0012^{\circ}$, corresponding to the grain size of $\sim 27 \mathrm{~nm}$ (calculated by using Scherrer formula). ${ }^{27}$ The effect of crystalline size and the strain on XRD peak broadening was further investigated by using Williamson-Hall (W-H) plot. ${ }^{28}$ The intercept of the linear fit gives the inverse of the crystalline size $\langle d\rangle_{\mathrm{XRD}} \sim 24 \mathrm{~nm}$ and slope of the curve gives strain $\eta \sim 8.341 \times 10^{-5}$. The obtained low value of crystalline size as compared to mean diameter indicates effect of annealing resulted in the formation of multi granular $\mathrm{Cr}_{2} \mathrm{O}_{3}$ nanorod. During annealing process, in the initial stage surface of $\mathrm{CrO}_{2}$
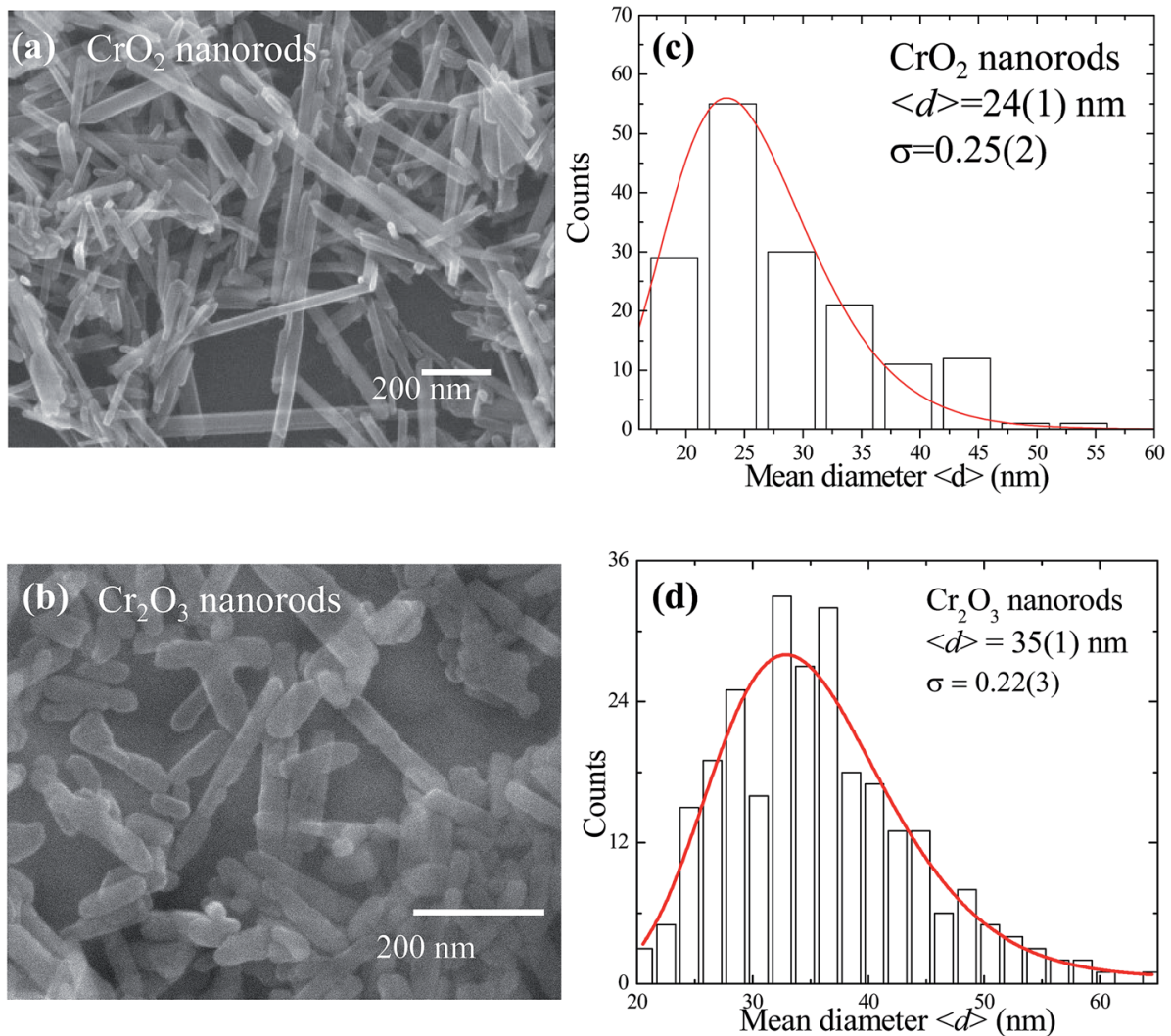

Fig. 1 (a) and (b) SEM images of $\mathrm{CrO}_{2}$ and $\mathrm{Cr}_{2} \mathrm{O}_{3}$ NRs. (c) and (d) Histogram of the mean diameter distribution of NRs where the solid line represents the fitting curve using log-normal distribution function. 

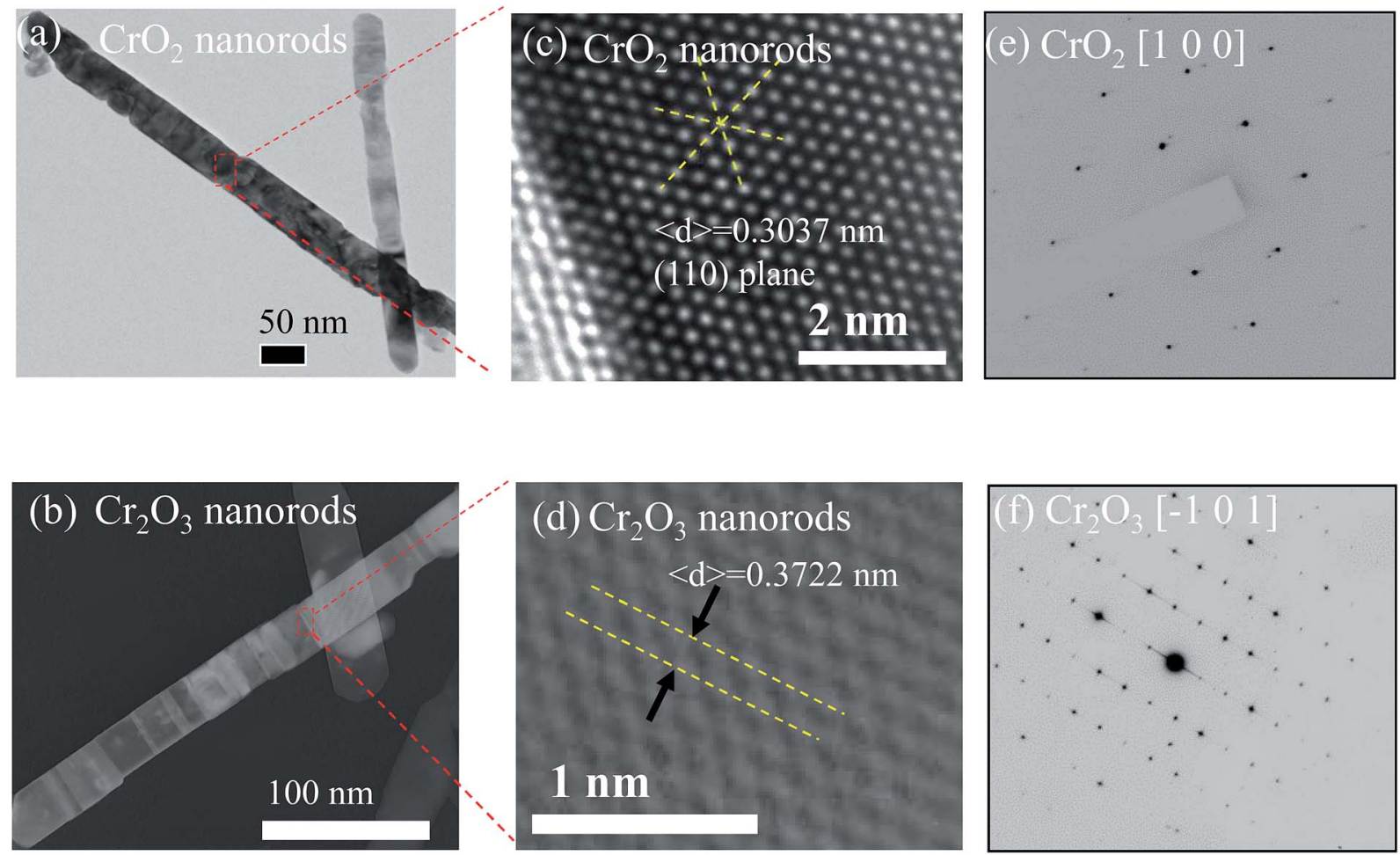

Fig. 2 (a) and (b) TEM, (c) and (d) HR-TEM, and (e) and (f) SAED patterns for $\mathrm{CrO}_{2}$ and $\mathrm{Cr}_{2} \mathrm{O}_{3} \mathrm{NRs}$, respectively.

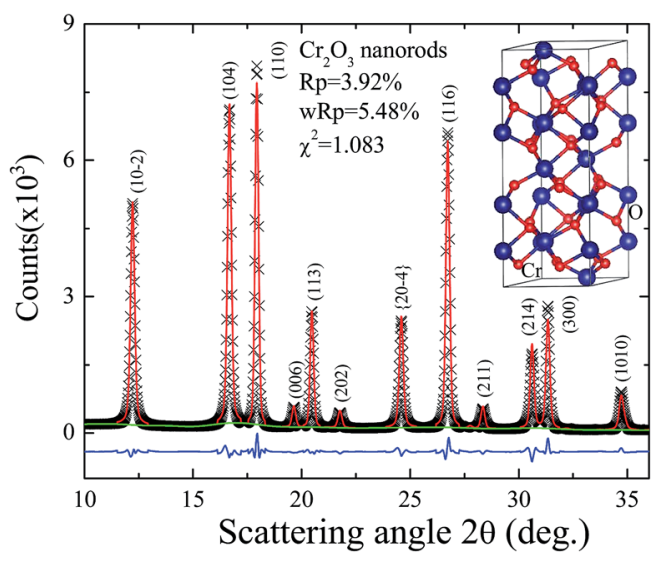

Fig. 3 X-ray diffraction and Rietveld refinement of $\mathrm{Cr}_{2} \mathrm{O}_{3} \mathrm{NRs}$.

nanorod oxidized to $\mathrm{Cr}_{2} \mathrm{O}_{3}$, resulting in the formation of nanoscale layer of $\mathrm{Cr}_{2} \mathrm{O}_{3}$ grains at the surface. Diffusion of oxygen into the $\mathrm{CrO}_{2}$ voids takes place through these microcracks, resulting in the growth of $\mathrm{Cr}_{2} \mathrm{O}_{3}$ at the grain boundaries which leads to the formation of multi-granular nanorod and hence size estimated from $\mathrm{W}-\mathrm{H}$ plot will be use in the further discussions. For estimating lattice parameters SR-XRD pattern was analyzed by refining spectra using Rietveld analysis ${ }^{29}$ with general structural analysis system (GSAS) software package. ${ }^{30}$ As shown in Fig. 3, the red solid line is the fitted curve to the diffraction pattern indicated by crosses. The green curve is fit to the background and blue curve in the bottom of figure gives the difference between observed and fitted pattern. The fitted values of lattice constant $a=b=4.9667 \AA$, and $c=13.6172 \AA$ confirm the $R \overline{3} c$ hexagonal $\mathrm{Cr}_{2} \mathrm{O}_{3}$ phase, as shown in the inset. The value of lattice constant and strain is very close to the value reported from $18 \mathrm{~nm} \mathrm{Cr}_{2} \mathrm{O}_{3}$ NPs. ${ }^{17}$ As compared to reported values from bulk $\mathrm{Cr}_{2} \mathrm{O}_{3}(a=b=4.9590 \AA$, and $c=13.5890 \AA)$, the observed lattice expansion from $\mathrm{Cr}_{2} \mathrm{O}_{3} \mathrm{NRs}$ is ascribed to finite size effect.

\subsection{Magnetic properties}

Fig. 4(a) shows the temperature $(T)$ dependence of magnetization $M(T)$ curve measured with ZFC-FC mode from 2 to $350 \mathrm{~K}$ with an applied magnetic field of $H_{\mathrm{a}}=100$ Oe. From $M(T)$ curves, with the increase of $T$ four clear features are seen. First, in low $T$ region an abrupt drop in the FC magnetization below $15 \mathrm{~K}$ assigned to surface frozen spins. Second, a blocking temperature $T_{\mathrm{B}}=30 \mathrm{~K}$ is defined as the maximum value of ZFC curve and could be related to a superparamagnetic (SPM) property of NRs. According to the Neel-Brown model ${ }^{31}$ the $T_{\mathrm{B}}$ for $\mathrm{Cr}_{2} \mathrm{O}_{3}$ NRs can be defined as $T_{\mathrm{B}} \approx K V / 22 k_{\mathrm{B}}$, where $K$ is the magnetocrystalline anisotropy energy density. Using $T_{\mathrm{B}}=30 \mathrm{~K}$, above equation yields $K=1.259 \times 10^{4} \mathrm{erg} \mathrm{cm}^{-3}$ for $24 \mathrm{~nm} \mathrm{Cr}_{2} \mathrm{O}_{3}$, which is one order smaller than the value reported from $24 \mathrm{~nm} \mathrm{Cr}_{2} \mathrm{O}_{3} \mathrm{NPs}^{17}$ Third, an irreversible temperature $T_{\mathrm{irr}} \sim 70 \mathrm{~K}$ is defined as the temperature at which $\left(M_{\mathrm{FC}}-M_{\mathrm{ZFC}}\right) / M_{\mathrm{FC}}(2 K) \approx 0.02$, relating to SG like behavior i.e. short-range spin-correlation. Fourth, above $T_{\text {irr }}$, SPM signals dominate, magnetization decreases with increase of $T$ and a plateau region around $T_{\mathrm{N}} \sim 295 \mathrm{~K}$ is observed. This value is very close to the bulk AF transition temperature $\sim 307 \mathrm{~K}$ and therefore corresponds to Neel temperature of $\mathrm{Cr}_{2} \mathrm{O}_{3}$ NRs. Fig. 4(b) shows the isothermal 

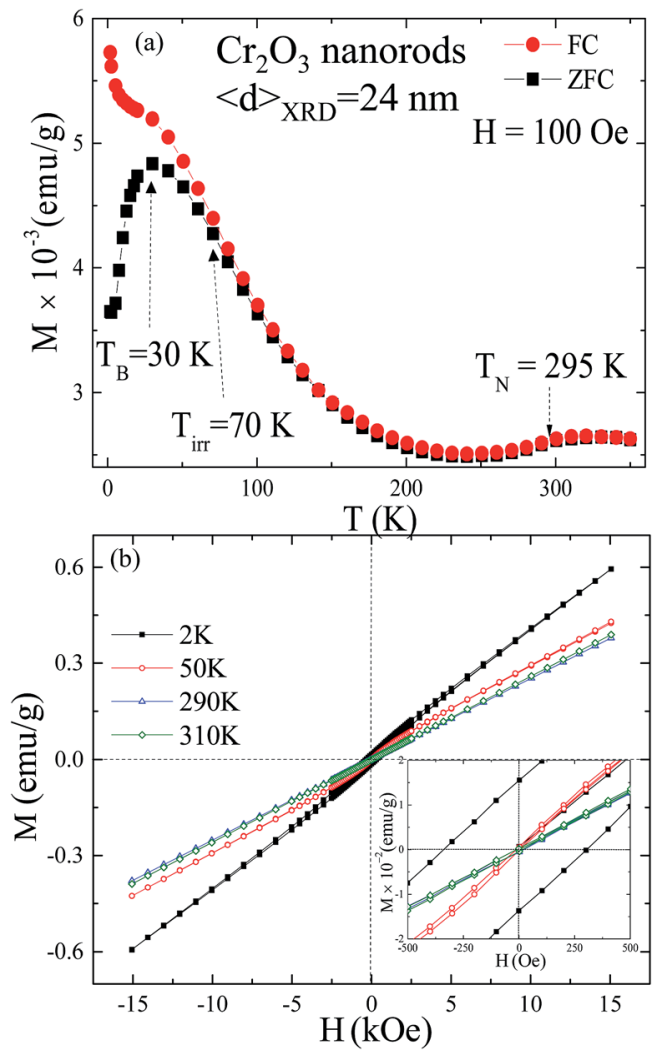

Fig. 4 (a) ZFC-FC magnetization curve of $\mathrm{Cr}_{2} \mathrm{O}_{3}$ NRs measured in a field of $100 \mathrm{Oe}$. (b) Magnetic hysteresis loop measured at 2, 50, 290 and $310 \mathrm{~K}$. Inset of (b) shows the near zero-field region of hysteresis loop.

magnetization loop $M(H)$ at selective temperatures 2, 50, 290 and $310 \mathrm{~K}$ measured with ZFC protocol over \pm 15 kOe field. The applied magnetic field dependence of the magnetization reveals different behaviors at various temperatures. At $T=310 \mathrm{~K}$, the magnetization presents a lineal dependence with the applied magnetic field in agreement with the paramagnetic (PM) state of the $\mathrm{Cr}^{3+}$ ions. At intermediate temperature of plateau region, $T_{\mathrm{B}}$ $<T<T_{\mathrm{N}}$, as shown in Fig. 4(a), the $M\left(H_{\mathrm{a}}\right)$ curves are reversible and reveal a curvature characteristic behavior of a superparamagnetic assembly of nanorods. At $2 \mathrm{~K}, M(H)$ loop exhibit a weak FM behavior, with linear and non-linear curve in highand low-field region having a coercivity of $H_{\mathrm{C}}=\left({H_{\mathrm{C}}}^{+}-\mathrm{H}_{\mathrm{C}}{ }^{-}\right) / 2=$ 320 Oe, as shown in the inset of Fig. 4(b). $\mathrm{H}_{\mathrm{C}}{ }^{+}$and $\mathrm{H}_{\mathrm{C}}{ }^{-}$are the field corresponding to the points in the forward and reverse branches of the $M(H)$ loop at which magnetization is zero. No noticeable difference in the $M(H)$ loop of 50, 290 and $310 \mathrm{~K}$ were found between the measurements made with a field-increasing and -decreasing loop suggesting PM/AF property above $T_{\mathrm{B}}$. Furthermore, value of magnetization at $310 \mathrm{~K}$ is slightly higher than at $290 \mathrm{~K}$ consistent with the observed plateau region around $T_{\mathrm{N}} \sim 295 \mathrm{~K}$ from $M(T)$ curve. The observed weak ferromagnetism at $2 \mathrm{~K}$ is could be due to uncompensated surface spins at the interface of crystalline domains.

Spontaneous exchange bias (SEB) results from a break in the symmetry across the FM-AFM interface and setting up of the UA during the first field of hysteresis loop measurement. ${ }^{21}$ To study the SEB phenomenon magnetization versus applied magnetic field, $M\left(H_{\mathrm{a}}\right)$ was measured using a p-type $\left(0 \rightarrow+H_{\max } \rightarrow-H_{\max }\right.$ $\left.\rightarrow+H_{\max }\right)$ protocol and n-type $\left(0 \rightarrow-H_{\max } \rightarrow+H_{\max } \rightarrow-H_{\max }\right)$ protocol in ZFC mode at $5 \mathrm{~K}$, as shown in Fig. 5(a). The non-zero value of $H_{\mathrm{C}}\left(H_{\mathrm{C}}=\left(\left|H_{\mathrm{C}}{ }^{+}\right|+\left|H_{\mathrm{C}}{ }^{-}\right|\right) / 2\right)$ and remanence $M_{\mathrm{r}}\left(M_{\mathrm{r}}=\right.$ $\left.\left(\left|M_{\mathrm{r}}{ }^{+}\right|+\left|{M_{\mathrm{r}}}^{-}\right|\right) / 2\right)$ clearly point to the existence of a weak-FM component at $5 \mathrm{~K} . M_{\mathrm{r}}{ }^{+}$and ${M_{\mathrm{r}}}^{-}$are the remanent magnetization corresponding to the points in the forward and reverse branches of the $M(H)$ loop at zero applied field. However, p- and n-type $M(H)$ loops at $40 \mathrm{~K}$ (above $T_{\mathrm{B}}$ ) are symmetric and exhibits PM property having zero value of $H_{\mathrm{C}}$ and $M_{\mathrm{r}}$, consistent with $M(T)$ curve. The obtained values of $H_{\mathrm{C}}$ with both p- and n-type protocol at different $T$ exhibits similar temperature dependencies and vanishes above $T_{\mathrm{B}}$ as depicted in Fig. 5(b). Similar temperature dependency was also observed for $M_{\mathrm{r}}$ as shown in the inset of Fig. 5(b). Furthermore, $M(H)$ loops in Fig. 5(a) are highly asymmetric and p-type (n-type) is shifted toward the negative (positive) magnetic axis. The $M(H)$ loop asymmetry along the applied magnetic field axis can be quantified as SEB, $H_{\mathrm{SEB}}=\left(\left|{H_{\mathrm{C}}}^{+}\right|-\left|{H_{\mathrm{C}}}^{-}\right|\right) / 2$. The estimated values of $H_{\mathrm{SEB}}$ with pand n-type $M(H)$ at $5 \mathrm{~K}$ shows similar value of -18 and $8.5 \mathrm{Oe}$, respectively. Slightly lower values of $H_{\mathrm{SEB}}$ are obtained after the n-type measurements than those of the p-type. Maity et al. ${ }^{32,33}$ reported $H_{\mathrm{SEB}}$ of -850 Oe and +615 Oe after p- and n-type hysteresis loop measurements from the $\mathrm{Bi}_{2} \mathrm{Fe}_{4} \mathrm{O}_{9}-\mathrm{BiFeO}_{3} \mathrm{FM} /$ AFM system, respectively. The slight difference observed in the behavior of SEB field obtained using the p- and n-type protocols is a common phenomenon. The reported high value of SEB from $\mathrm{Bi}_{2} \mathrm{Fe}_{4} \mathrm{O}_{9}-\mathrm{BiFeO}_{3}$ as compared to $\mathrm{Cr}_{2} \mathrm{O}_{3}$ NRs is due to the inter-coupling between the log-range ordered FM and AFM spins at the interface. ${ }^{32,33}$ Furthermore, the p-type and ntype $M\left(H_{\mathrm{a}}\right)$ loops measured in the ZFC mode are almost symmetric in nature and exhibit a similar value for SEB, which is within the experimental measurement error.

The observed similar value of $H_{\mathrm{SEB}}$ in both field direction indicate shifts in $M(H)$ loops are not experimental artifacts, but rather an intrinsic property of $\mathrm{Cr}_{2} \mathrm{O}_{3}$ NRs. The temperature dependency of $H_{\text {SEB }}$ obtained from both p- and n-type $M(H)$ loops vanishes at and above $T_{\mathrm{B}}$ as shown in Fig. 5(c). To further confirm the SEB loop shift, FC $M(H)$ loops were measured with a cooling field of $\pm 10 \mathrm{kOe}$ at $5 \mathrm{~K}$ shown in Fig. 5(d) reveals an enhanced asymmetric behavior with loop shift in opposite field directions. The estimated values of $H_{\mathrm{C}}, M_{\mathrm{r}}$ and $H_{\mathrm{EB}}$ for cooling field of $+10 \mathrm{kOe}(-10 \mathrm{kOe})$ are $213 \mathrm{Oe}(225 \mathrm{Oe}), 0.011 \mathrm{emu} \mathrm{g}^{-1}$ $\left(0.012 \mathrm{emu}^{-1}\right)$ and -125 Oe (89 Oe), respectively. The above observed results confirm the existence of UA below $T_{\mathrm{B}}$ at the interface of frustrated surface spins and the uncompensated $\mathrm{AF}$ core. Furthermore, this is first report to observe SEB in an AF $\mathrm{Cr}_{2} \mathrm{O}_{3}$ NRs. The observed non-zero values of $M_{\mathrm{r}}$ and $H_{\mathrm{C}}$ confirm the $\mathrm{FM}$ behavior of the $24 \mathrm{~nm} \mathrm{Cr}_{2} \mathrm{O}_{3}$ nanorods originating from finite size effect. It is worthy to note that the observed minor loop of the magnetization at low temperature may originate from the non-saturated magnetization at higher applied magnetic field, causing the vertical/horizontal shift of magnetization. In the present study, such weak magnetic properties can be explained by the presence of uncompensated spins at the 


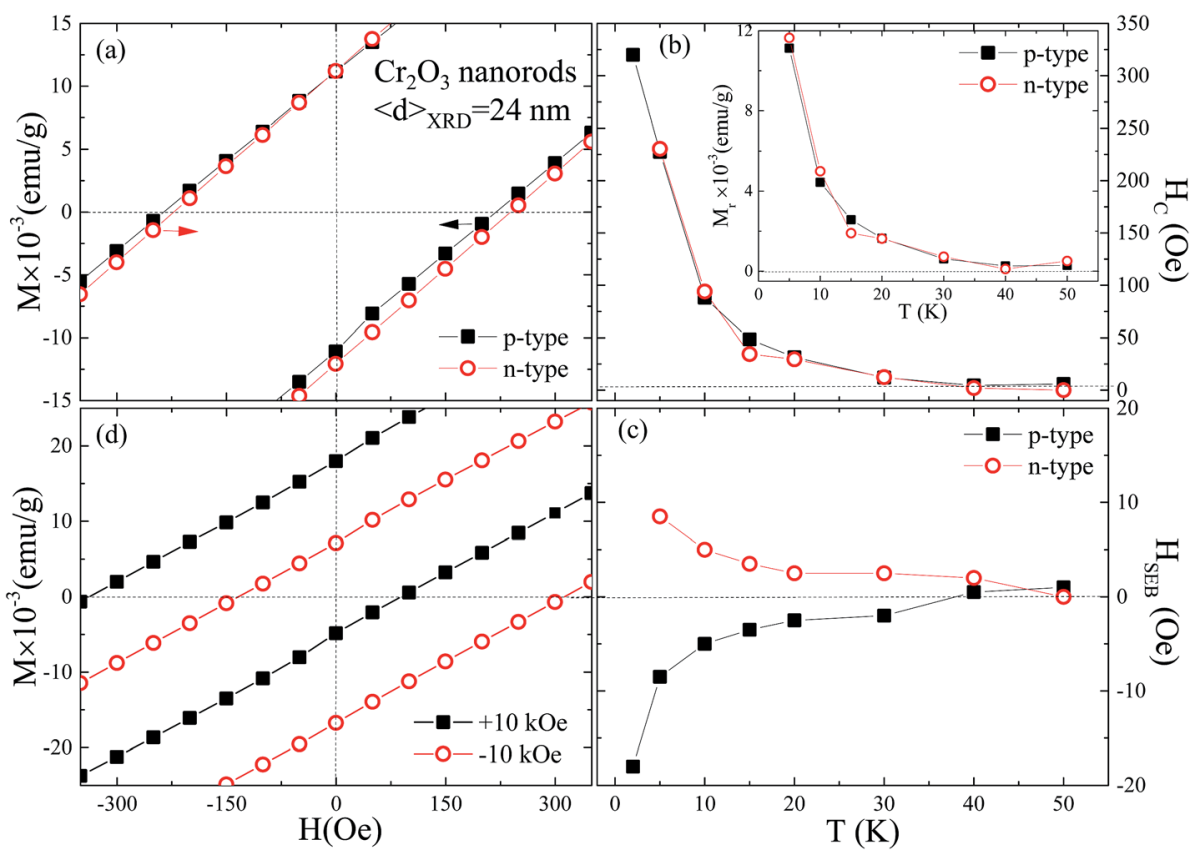

Fig. 5 (a) Near zero-field region of magnetic hysteresis loop measured at $5 \mathrm{~K}$ with $\mathrm{p}$ - and $\mathrm{n}$-type protocols after zero-field cooling. Temperature dependency of (b) coercivity and (c) SEB field obtained from p-and n-type of hysteresis loops. Inset of (b) shows the temperature dependency of remenance. (d) Near zero-field region of magnetic hysteresis loop measured at $5 \mathrm{~K}$ with field cooling in $\pm 10 \mathrm{kOe}$.

surface of $\mathrm{Cr}_{2} \mathrm{O}_{3}$ nanorods, as has been discussed in previous reports for $\mathrm{Cr}_{2} \mathrm{O}_{3}$ nanoparticles. ${ }^{34-37}$ In nanoscale materials, the uncompensated surface spins show a lower coordination number, resulting a net magnetic moment can be induced on the surface. ${ }^{38,39}$ The observed SEB effect arises from intercoupling between the short-range ordered clusters of frustrated surface spins and uncompensated antiferromagnetic cores at the interface during the first field of hysteresis loop measurement. Such inter-coupling sets up unidirectional anisotropy at the interface; for a detailed description was discussed in our previous work. $^{23}$

\subsection{Memory effect}

The magnetic anisotropy plays an important role in shaping the magnetic properties of nanoscale material which can be altered by the random size distribution, strong inter particle interactions and unidirectional anisotropy in the core/shell magnetic nanoparticles. ${ }^{21,40}$ The effect of enhanced magnetic anisotropy on the magnetic properties of nanoparticles can be studied effectively by measuring the thermoremanent spin dynamics ${ }^{41}$ the memory effect ${ }^{42}$ and the effect of aging ${ }^{43}$ using field cooling and zero field cooling processes. The field cooling and zero field cooling memory effects have been used for the characterization of superparamagnetic ${ }^{44}$ and spin-glass ${ }^{42}$ systems. To characterize magnetic properties, the memory effect in FC magnetization with FC protocol is investigated. The results for FC memory effect within 2 to $50 \mathrm{~K}$ temperature range are depicted in Fig. 6. Initially NRs were cool down from $300 \mathrm{~K}$ down to $2 \mathrm{~K}$ with a cooling rate of $2 \mathrm{~K} \mathrm{~min}^{-1}$ in a static magnetic field of 100 Oe and magnetization recorded as a function of temperature. During recording, temperature of the sample was "held" at $40,30,20$, and $10 \mathrm{~K}$ for a time period of $5 \mathrm{~h}$ each and magnetic moments were allowed to relax by switching off the magnetic field. After $5 \mathrm{~h}$ relaxation, magnetic field turn on and measurement was subsequently resumed with the same cooling rate. The above magnetization curve is designated as "cooling curve" shown in Fig. 6. After reaching the desired low temperature of $2 \mathrm{~K}$, NRs were warmed and magnetization recorded at rate of $2 \mathrm{~K} \mathrm{~min}^{-1}$ in 100 Oe field without any stop, designated as "warming" curve, also called as "memory curve" as shown in Fig. 6. Discernible evidence of steps is clearly visible in the memory curve around 10,20 and $30 \mathrm{~K}$ below $T_{\mathrm{B}}$. The observed step-like behavior indicates that the spin configuration imprinted in the blocking state (below $T_{\mathrm{B}}$ ) of NRs is retrieved by the memory curve. This step like behavior is the

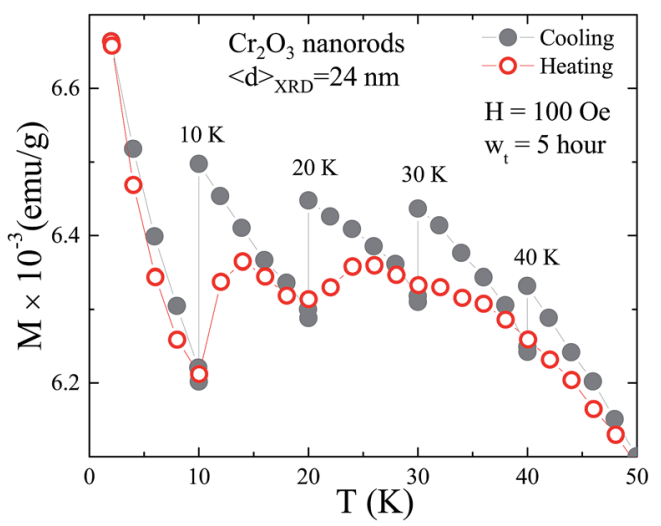

Fig. 6 FC memory effect in the 100 Oe field with a halt of $5 \mathrm{~h}$ at 10,20 , 30 and $40 \mathrm{~K}$. 
signature of the magnetic memory effect which can be used as a good measure for understanding SPM or SG system. The unique part in Fig. 6 is the increasing behavior of magnetization with the decrease of temperature which has been used as a fingerprint test to distinguish SG and blocked SPM system. In non-interacting SPM nanostructure moments prefer to stay blocked along the easy axis direction which is along the field direction resulting in increase of magnetization with the decrease of temperature. On the other hand, an interacting SG system shows decrease in the magnetization with the decrease of temperature. Therefore, observed FC memory effect is could be either because of wide distribution in length of NRs as observed from SEM images causing distribution in magnetocrystalline anisotropy or because of setup of UA in blocked state. In a spin glassy system, the length of the spin-spin correlation grows during the stop, even in a zero field, and a memory dip typically shows up upon reheating. This is not possible in a non/weak-interacting nanoparticle system which does not show the memory dip in the ZFC mode. The above finding indicates that the FC memory effect in stronglyinteracting $24 \mathrm{~nm} \mathrm{Cr}_{2} \mathrm{O}_{3} \mathrm{NRs}$ could be induced either by the size distribution (which resulted in the broad distribution of blocking temperatures) or the strongly pinned interfacial spin mediated UA. However, the observed decreasing behavior of both the FC memory effect and the SEB field with the increase of temperature indicates that the memory effect in $\mathrm{Cr}_{2} \mathrm{O}_{3} \mathrm{NRs}$ is mediated by strongly pinned interfacial spins. However, size distribution leads to broad distribution of $T_{\mathrm{B}}$, which is not consistent with $M(T)$. Furthermore, observed non-zero coercivity in blocked state indicate weak ferromagnetic behavior which differs significantly from SPM system. Therefore, we confirmed that observed memory effect from AF NRs could be manifested by UA of $\mathrm{Cr}_{2} \mathrm{O}_{3}$.

\section{Conclusion}

The AF $\mathrm{Cr}_{2} \mathrm{O}_{3}$ nanorods (NRs) with diameter of $\langle d\rangle_{\mathrm{XRD}}=24 \mathrm{~nm}$ are investigated by means of DC magnetometer in order to study the unidirectional anisotropy (UA) properties. Temperature dependent magnetization $M(T)$ reveal $T_{\mathrm{B}}=30 \mathrm{~K}$ below which system is in blocked state. A SEB field of 18 Oe is reported at $2 \mathrm{~K}$ attributed to setting up of UA during the first field of hysteresis loop measurement. The field-cooled (FC) magnetization relaxation measured at different temperature shows the presence of multimagnetic anisotropy. However, disappearance of FC memory effect, coercivity and SEB field above $T_{\mathrm{B}}$ and observed narrow distribution of $M(T)$ curve rule out the possibility of interacting SPM system. Hence, we conclude that FC memory effect from $\mathrm{AF} \mathrm{Cr}_{2} \mathrm{O}_{3}$ NRs is mediated by UA.

\section{Acknowledgements}

We would like to thank the Ministry of Science and Technology (MOST) of the Republic of China for their financial support of this research through project number MOST-105-2112-M-259003.

\section{References}

1 A. Asenjo-Garcia, J. D. Hood, D. E. Chang and H. J. Kimble, Atom-light interactions in quasi-one-dimensional nanostructures: a Green's-function perspective, Phys. Rev. A, 2017, 95(3), 033818.

2 Y. Xia, P. Yang, Y. Sun, Y. Wu, B. Mayers, B. Gates, Y. Yin, F. Kim and H. Yan, One-Dimensional Nanostructures: Synthesis, Characterization, and Applications, Adv. Mater., 2003, 15(5), 353-389.

3 A. C. Gandhi, H.-J. Hung, P.-H. Shih, C.-L. Cheng, Y.-R. Ma and S. Y. Wu, In Situ Confocal Raman Mapping Study of a Single Ti-Assisted ZnO Nanowire, Nanoscale Res. Lett., 2009, 5(3), 581-586.

4 R. S. Devan, R. A. Patil, J.-H. Lin and Y.-R. Ma, OneDimensional Metal-Oxide Nanostructures: Recent Developments in Synthesis, Characterization, and Applications, Adv. Funct. Mater., 2012, 22(16), 3326-3370.

5 S. Yang, S. Liu, C. Lan and S. Yang, Single crystalline $\mathrm{Cr}_{2} \mathrm{O}_{3}$ nanowires/nanobelts: $\mathrm{CrCl}_{3}$ assistant synthesis and novel magnetic properties, Appl. Surf. Sci., 2012, 258(22), 8965-8969.

6 J. L. Xie, C. X. Guo and C. M. Li, Construction of onedimensional nanostructures on graphene for efficient energy conversion and storage, Energy Environ. Sci., 2014, $7(8), 2559-2579$.

7 T. Zhai and J. Yao, One-Dimensional Nanostructures, Principles and Applications, John Wiley \& Sons, Inc., 2012.

8 A. C. Gandhi, T. S. Chan, J. Pant and S. Y. Wu, Strong PinnedSpin-Mediated Memory Effect in NiO Nanoparticles, Nanoscale Res. Lett., 2017, 12(1), 207.

9 D. Tobia, E. L. Winkler, R. D. Zysler, M. Granada and H. E. Troiani, Superparamagnetism in AFM $\mathrm{Cr}_{2} \mathrm{O}_{3}$ nanoparticles, J. Alloys Compd., 2010, 495(2), 520-523.

10 D. Tobia, E. De Biasi, M. Granada, H. E. Troiani, G. Zampieri, E. Winkler and R. D. Zysler, Evolution of the magnetic anisotropy with particle size in antiferromagnetic $\mathrm{Cr}_{2} \mathrm{O}_{3}$ nanoparticles, J. Appl. Phys., 2010, 108(10), 104303.

11 (a) L. M. Corliss, J. M. Hastings, R. Nathans and G. Shirane, Magnetic Structure of $\mathrm{Cr}_{2} \mathrm{O}_{3}$, J. Appl. Phys., 1965, 36(3), 10991100; (b) P. J. Brown, J. B. Forsyth, E. Lelièvre-Berna and F. Tasset, Determination of the magnetization distribution in $\mathrm{Cr}_{2} \mathrm{O}_{3}$ using spherical neutron polarimetry, J. Phys.: Condens. Matter, 2002, 14(8), 1957.

12 S. A. Makhlouf, Z. H. Bakr, H. Al-Attar and M. S. Moustafa, Structural, morphological and electrical properties of $\mathrm{Cr}_{2} \mathrm{O}_{3}$ nanoparticles, J. Mater. Sci. Eng. B, 2013, 178(6), 337-343.

13 S. Sahoo and C. Binek, Piezomagnetism in epitaxial $\mathrm{Cr}_{2} \mathrm{O}_{3}$ thin films and spintronic applications, Philos. Mag. Lett., 2007, 87(3-4), 259-268.

14 T. R. McGuire, E. J. Scott and F. H. Grannis, Antiferromagnetism in a $\mathrm{Cr}_{2} \mathrm{O}_{3}$ Crystal, Phys. Rev., 1956, 102(4), 1000-1003.

15 D. Tobia, E. Winkler, R. D. Zysler, M. Granada and H. E. Troiani, Size dependence of the magnetic properties 
of antiferromagnetic $\mathrm{Cr}_{2} \mathrm{O}_{3}$ nanoparticles, Phys. Rev. B: Condens. Matter Mater. Phys., 2008, 78(10), 104412.

16 D. Li, Z. Han, J. G. Zheng, X. L. Wang, D. Y. Geng, J. Li and Z. D. Zhang, Spin canting and spin-flop transition in antiferromagnetic $\mathrm{Cr}_{2} \mathrm{O}_{3}$ nanocrystals, J. Appl. Phys., 2009, 106, 053913.

17 P. Hajra, P. Brahma, S. Dutta, S. Banerjee and D. Chakravorty, Enhancement of magnetic anisotropy in mechanically attrited $\mathrm{Cr}_{2} \mathrm{O}_{3}$ nanoparticles, J. Magn. Magn. Mater., 2012, 324(7), 1425-1430.

18 O. Gomonay, S. Kondovych and V. Loktev, Shape-induced anisotropy in antiferromagnetic nanoparticles, J. Magn. Magn. Mater., 2014, 354, 125-135.

19 M. Sasaki, P. E. Jönsson, H. Takayama and H. Mamiya, Aging and memory effects in superparamagnets and superspin glasses, Phys. Rev. B: Condens. Matter Mater. Phys., 2005, 71(10), 104405.

20 K. Jonason, E. Vincent, J. Hammann, J. P. Bouchaud and P. Nordblad, Memory and Chaos Effects in Spin Glasses, Phys. Rev. Lett., 1998, 81(15), 3243-3246.

21 A. C. Gandhi, P. M. Reddy, T.-S. Chan, Y.-P. Ho and S. Y. Wu, Memory effect in weakly-interacting $\mathrm{Fe}_{3} \mathrm{O}_{4}$ nanoparticles, RSC Adv., 2015, 5(103), 84782-84789.

22 M. Vasilakaki, K. N. Trohidou, D. Peddis, D. Fiorani, R. Mathieu, M. Hudl, P. Nordblad, C. Binns and S. Baker, Memory effects on the magnetic behavior of assemblies of nanoparticles with ferromagnetic core/antiferromagnetic shell morphology, Phys. Rev. B: Condens. Matter Mater. Phys., 2013, 88(14), 140402.

23 A. C. Gandhi, J. Pant and S. Y. Wu, Dense inter-particle interaction mediated spontaneous exchange bias in $\mathrm{NiO}$ nanoparticles, $R S C A d v$., 2016, 6(3), 2079-2086.

24 T. P. Kobylinski and B. W. Taylor, The catalytic chemistry of nitric oxide: I. The effect of water on the reduction of nitric oxide over supported chromium and iron oxides, J. Catal., 1973, 31, 450.

25 M. Audier, M. Salaün, H. Roussel, F. Delyon and M. Duneau, CrO2-to-Cr2O3 Transformation in a Three-Dimensional Interference Field of Ultraviolet Laser Light, Cryst. Growth Des., 2010, 10, 1923-1928.

26 W.-Q. Han, L. Wu, A. Stein, Y. Zhu, J. Misewich and J. Warren, Oxygen-Deficiency-Induced Superlattice Structures of Chromia Nanobelts, Angew. Chem., Int. Ed., 2006, 45, 6554 .

27 B. E. Warren, X-ray Diffraction, Addsion-Wesley, Reading, MA, 1969.

28 A. C. Gandhi, J. Pant, S. D. Pandit, S. K. Dalimbkar, T.-S. Chan, C.-L. Cheng, Y.-R. Ma and S. Y. Wu, ShortRange Magnon Excitation in NiO Nanoparticles, J. Phys. Chem. C, 2013, 117(36), 18666-18674.

$29 \mathrm{H}$. Rietveld, A profile refinement method for nuclear and magnetic structures, J. Appl. Crystallogr., 1969, 2(2), 65-71.

30 A. C. Larson and R. B. Von Dreele Los, Alamos National Laboratory Report LA-UR-86-748 Los Alamos, Los Alamos National Laboratory, 1987.
31 (a) L. Neel, Théorie du traînage magnétique des ferromagnétiques en grains fins avec application aux terres 5 cuites, Ann. Geophys., 1949, 5(37), 99-136; (b) W. F. Brown, Thermal Fluctuations of a Single-Domain Particle, Phys. Rev., 1963, 130(5), 1677-1686.

32 T. Maity, S. Goswami, D. Bhattacharya, G. C. Das and S. Roy, Spontaneous exchange bias in a nanocomposite of $\mathrm{BiFeO}_{3}{ }^{-}$ $\mathrm{Bi}_{2} \mathrm{Fe}_{4} \mathrm{O}_{9}$, J. Appl. Phys., 2013, 113, $17 \mathrm{D} 916$.

33 T. Maity, S. Goswami, D. Bhattacharya and S. Roy, Superspin Glass Mediated Giant Spontaneous Exchange Bias in a Nanocomposite of $\mathrm{BiFeO}_{3}-\mathrm{Bi}_{2} \mathrm{Fe}_{4} \mathrm{O}_{9}$, Phys. Rev. Lett., 2013, 110, 107201.

34 M. Bañobre-López, C. Vázquez-Vázquez, J. Rivas and M. A. López-Quintela, Magnetic properties of chromium (III) oxide nanoparticles, Nanotechnology, 2003, 14, 318-322.

35 W. S. Zhang, E. Brueck, Z. D. Zhang, O. Tegus, W. F. Li, P. Z. Si, D. Y. Geng and K. H. J. Buschow, Structure and magnetic properties of $\mathrm{Cr}$ nanoparticles and $\mathrm{Cr}_{2} \mathrm{O}_{3}$ nanoparticles, J. Phys. B: At., Mol. Opt. Phys., 2005, 358, 332-338.

36 C. Vázquez-Vázquez, M. Bañobre-López, M. A. LópezQuintela, L. E. Hueso and J. Rivas, Evidence of weak ferromagnetism in chromium(III) oxide particles, J. Magn. Magn. Mater., 2004, 272-276, 1547-1548.

37 S. A. Makhlouf, Magnetic properties of $\mathrm{Cr}_{2} \mathrm{O}_{3}$ Nanoparticles, J. Magn. Magn. Mater., 2004, 272-276, 1530-1532.

38 M. Feygenson, A. Kou, L. E. Kreno, A. L. Tiano, J. M. Patete, F. Zhang, M. S. Kim, V. Solovyov, S. S. Wong and M. C. Aronson, Properties of highly crystalline $\mathrm{NiO}$ and $\mathrm{Ni}$ nanoparticles prepared by high-temperature oxidation and reduction, Phys. Rev. B: Condens. Matter Mater. Phys., 2010, 81, 014420.

39 A. C. Santulli, M. Feygenson, F. E. Camino, M. C. Aronson and S. S. Wong, Synthesis and Characterization of OneDimensional Cr2O3 Nanostructures, Chem. Mater., 2011, 23, 1000-1008.

40 G. Zhang, K. Potzger, S. Zhou, A. Mücklich, Y. Ma and J. Fassbender, Memory effect of magnetic nanoparticle systems originating from particle size distribution, $\mathrm{Nucl}$. Instrum. Methods Phys. Res., Sect. B, 2009, 267(8-9), 15961599.

41 M. Ulrich, J. García-Otero, J. Rivas and A. Bunde, Slow relaxation in ferromagnetic nanoparticles: indication of spin-glass behavior, Phys. Rev. B: Condens. Matter Mater. Phys., 2003, 67(2), 024416.

42 K. Jonason, E. Vincent, J. Hammann, J. P. Bouchaud and P. Nordblad, Memory and chaos effects in spin glasses, Phys. Rev. Lett., 1998, 81(15), 3243-3246.

43 T. Jonsson, J. Mattsson, C. Djurberg, F. A. Khan, P. Nordblad and P. Svedlindh, Aging in a magnetic particle system, Phys. Rev. Lett., 1995, 75(22), 4138-4141.

44 M. Sasaki, P. E. Jönsson, H. Takayama and H. Mamiya, Aging and memory effects in superparamagnets and superspin glasses, Phys. Rev. B: Condens. Matter Mater. Phys., 2005, 71(10), 104405. 